\title{
COMMENTARY
}

\section{Modeling human brain development with cerebral organoids}

\author{
Luca Muzio ${ }^{1,2^{*}}$ and G Giacomo Consalez
}

\begin{abstract}
The recent discovery of a new three-dimensional culture system for the derivation of cerebral organoids from human induced pluripotent stem cells provides developmental neurobiologists with the first example of a three-dimensional framework for the study of human brain development. This innovative approach permits the in vitro assembly of a human embryonic brain rudiment that recapitulates the developing human cerebrum. Organoids contain progenitor populations that develop to yield mature cortical neuron subtypes, potentially allowing investigators to study complex brain diseases that lack appropriate animal models.
\end{abstract}

The study of developmental neurogenesis, neuronal migration, circuit formation and synaptogenesis has led to substantial advances in our understanding of the signaling pathways and molecular mechanisms recruited in the course of normal brain development, and disrupted in its pathological aberrations. Mice and other animal models have played a crucial part in this context, allowing the molecular dissection of developmental neurogenesis. However, some notable features of the brain, such as the dramatic tangential expansion of the neocortex, reach their peak in humans, and distinguish Homo sapiens from other mammalian species. Therefore, some aspects unique to human neurogenesis make the mouse a suboptimal tool for the task. Moreover, while the genetic manipulation of the mouse genome has become extremely efficient and sophisticated, in many instances a direct analysis of human tissues bearing disease-causing mutations would be preferable to the study of a rodent model. A classic example is provided by neuronal migration defects leading to a smooth, lissencephalic cortex

\footnotetext{
* Correspondence: muzio.luca@hsr.it

'Division of Neuroscience, San Raffaele Scientific Institute, Via Olgettina 58, 20132, Milan, Italy

${ }^{2}$ Institute of Experimental Neurology (INSPE), San Raffaele Scientific Institute, Via Olgettina 58, 20132, Milan, Italy
}

devoid of sulci and gyri due to a massive decrease in overall neocortical surface. These disorders cannot be modeled efficiently in the mouse, a species that in normal conditions features a smooth cortex.

A groundbreaking paper published recently by Lancaster and coworkers begins to tackle this complex issue by proposing a homospecific model system to approach the biology of human neurogenesis and its genetic defects [1]. Starting from induced pluripotent stem (iPS) cells derived from healthy individuals, Lancaster and colleagues report the generation and analysis of three-dimensional tissue cultures of the developing human brain, dubbed cerebral organoids. These structures share many key features with the pattern and cytoarchitecture of the embryonic human brain. The single most radical innovation introduced by Lancaster and colleagues is the culture system that they adopt to grow organoids. Compared with the widely used protocol currently in use in many laboratories to grow neuroectodermal structures from iPS cells $[2,3]$, they develop a three-dimensional system based on a gel matrix that provides a scaffold for organogenesis and histogenesis. Although organoids plateau in size after 2 months, possibly due to suboptimal oxygen and nutrient uptake, both germinal niches and the post-mitotic cortical plate that form in these three-dimensional cultures contain cells exhibiting morphological and neurochemical features of radial glia and maturing neurons, respectively. Specifically, the molecular repertoire of radial glia is properly represented, as shown by the tissue distribution of several key transcription factors. In brain organoids, apical progenitors fully recapitulate interkinetic nuclear migration. Some of them produce outer radial glia, a progenitor population abundant in primates and poorly represented in the developing rodent brain [4]. Similarly, neurons of the cortical plate are correctly specified, as indicated both by the expression of appropriate markers and by the ability of these cells to display spontaneous $\mathrm{Ca}^{2+}$ surges. Brain organoids exhibit a physiological cortical plate lamination, probably orchestrated by Cajal-Retzius cells, which migrate to colonize the cortical marginal zone. 
Interestingly, in the same article Lancaster and colleagues further support the worth of their method by establishing organoids from iPS cells derived from microcephalic patients. In keeping with the neuropathology of microcephaly, iPS cells derived from patients carrying a truncating mutation in the CDK5RAP2 gene [5] generate mini brains that grow smaller than their wildtype controls. In addition, these mutant brain organoids yield a plethora of early differentiating neurons, in keeping with the notion that, in some forms of microcephaly, a dysregulated timing of cell differentiation causes the proliferating progenitor pool to be prematurely depleted.

This model provides a promising and novel tool to investigate cell-cell interactions and asks questions pertaining to cell autonomy in normal development and neurodevelopmental disorders. In so doing, organoids also provide a conceptual and practical alternative to the use of animal models. Cerebral organoids represent a powerful approach to answer questions relevant to cortical development and evolution, allowing one to perform heterochronic, heterotopic or interspecific grafts into a human cortical primordium. Strikingly, from a regenerative medicine perspective, organoids may also facilitate the isolation of solid grafts and dissociated neuronal precursors for autologous transplantation, particularly with regard to those cell types that are extremely difficult to generate in the artifactual environment of a Petri dish.

Obviously, at this stage, some extant limitations of this method are likely to preclude its use in certain applications. For example, organoids represent early time points in brain development, while later developmental stages featuring neuronal connectivity and the deployment of higher neural network functions cannot be adequately recapitulated in these structures. Furthermore, the differentiation of brain microvasculature is absent from organoids. Likewise, microglia cells - which originate from primitive myeloid progenitors arising at early developmental stages [6] - do not contribute to these iPSderived models. This omission may pose problems, since microglia have been proposed to regulate neurogenesis by modulating the size of the neural precursor pool [7].

In summary, this tantalizing technology will provide scientists with a purely neuronal and neuroglial model for the study of mutual interactions occurring between different neuroectodermal progenitors during human brain development (the radial glial unit). Moreover, organoids will boost the elaboration of new paradigms and strategies in regenerative medicine. Conversely, the analysis of more complex interactions that involve an interplay between neurons and non-neuronal cells will probably require the generation of a more advanced setup.

\section{Competing interests}

The authors declare that they have no competing interests.

Published: 24 Dec 2013

\section{References}

1. Lancaster MA, Renner M, Martin CA, Wenzel D, Bicknell LS, Hurles ME, Homfray T, Penninger JM, Jackson AP, Knoblich JA: Cerebral organoids model human brain development and microcephaly. Nature 2013, 501:373-379.

2. Soldner F, Jaenisch R: Medicine. iPSC disease modeling. Science 2012, 338:1155-1156.

3. Soldner F, Hockemeyer D, Beard C, Gao Q, Bell GW, Cook EG, Hargus G, Blak A, Cooper O, Mitalipova M, Isacson O, Jaenisch R: Parkinson's disease patient-derived induced pluripotent stem cells free of viral reprogramming factors. Cell 2009, 136:964-977.

4. Wang X, Tsai JW, LaMonica B, Kriegstein AR: A new subtype of progenitor cell in the mouse embryonic neocortex. Nat Neurosci 2011, 14:555-561.

5. Bond J, Roberts E, Springell K, Lizarraga SB, Scott S, Higgins J, Hampshire DJ, Morrison EE, Leal GF, Silva EO, Costa SM, Baralle D, Raponi M, Karbani G, Rashid Y, Jafri H, Bennett C, Corry P, Walsh CA, Woods CG: A centrosomal mechanism involving CDK5RAP2 and CENPJ controls brain size. Nat Genet 2005, 37:353-355.

6. Ginhoux F, Greter M, Leboeuf M, Nandi S, See P, Gokhan S, Mehler MF, Conway SJ, Ng LG, Stanley ER, Samokhvalov IM, Merad M: Fate mapping analysis reveals that adult microglia derive from primitive macrophages. Science 2010, 330:841-845.

7. Cunningham CL, Martinez-Cerdeno V, Noctor SC: Microglia regulate the number of neural precursor cells in the developing cerebral cortex. $J$ Neurosci 2013, 33:4216-4233.

\section{$10.1186 /$ scrt384}

Cite this article as: Muzio and Consalez: Modeling human brain development with cerebral organoids. Stem Cell Research \& Therapy 2013, $4: 154$ 